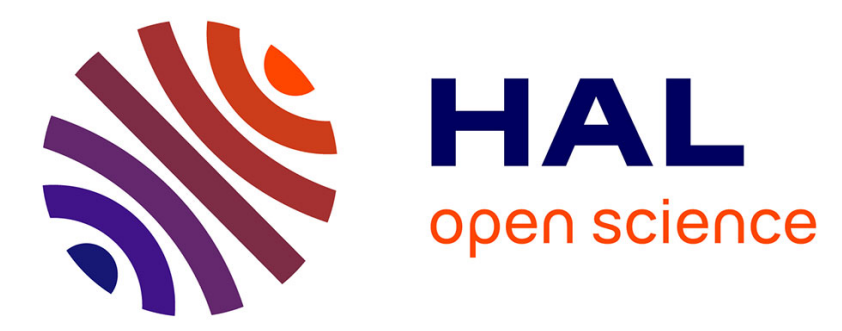

\title{
Electrical detection of light helicity using a quantum-dot-based hybrid device at zero magnetic field
}

F Cadiz, D Lagarde, B Tao, J Frougier, B Xu, X Devaux, S Migot, Z G Wang, X F Han, J-M George, et al.

\section{- To cite this version:}

F Cadiz, D Lagarde, B Tao, J Frougier, B Xu, et al.. Electrical detection of light helicity using a quantum-dot-based hybrid device at zero magnetic field. Physical Review Materials, 2020, 4, 10.1103/physrevmaterials.4.124603 . hal-03417725

\section{HAL Id: hal-03417725 \\ https://hal.science/hal-03417725}

Submitted on 5 Nov 2021

HAL is a multi-disciplinary open access archive for the deposit and dissemination of scientific research documents, whether they are published or not. The documents may come from teaching and research institutions in France or abroad, or from public or private research centers.
L'archive ouverte pluridisciplinaire HAL, est destinée au dépôt et à la diffusion de documents scientifiques de niveau recherche, publiés ou non, émanant des établissements d'enseignement et de recherche français ou étrangers, des laboratoires publics ou privés. 


\title{
Electrical detection of light helicity using a quantum-dot-based hybrid device at zero magnetic field
}

\author{
F. Cadiz, ${ }^{1}$ D. Lagarde, ${ }^{1}$ B. Tao, ${ }^{2}$ J. Frougier $\odot,{ }^{3}$ B. Xu,${ }^{4}$ X. Devaux, ${ }^{2}$ S. Migot, ${ }^{2}$ Z. G. Wang, ${ }^{4}$ X. F. Han, ${ }^{5}$ J.-M. George, ${ }^{3}$ \\ H. Carrere $\odot,{ }^{1}$ A. Balocchi, ${ }^{1}$ T. Amand, ${ }^{1}$ X. Marie, ${ }^{1}$ B. Urbaszek, ${ }^{1}$ H. Jaffrès $\odot,{ }^{3}$ Y. Lu $\odot,{ }^{2,}{ }^{*}$ and P. Renucci ${ }^{1, \dagger}$ \\ ${ }^{1}$ Université de Toulouse, INSA-CNRS-UPS, LPCNO, 135 Av. Rangueil, 31077 Toulouse, France \\ ${ }^{2}$ Institut Jean Lamour, Université de Lorraine, CNRS UMR7198, Campus ARTEM, 2 Allée André Guinier, BP 50840, 54011 Nancy, France \\ ${ }^{3}$ Unité Mixte de Physique, CNRS, Thales, Université Paris-Saclay, 91767 Palaiseau, France \\ ${ }^{4}$ Key Laboratory of Semiconductor Materials Science, Institute of Semiconductors, Chinese Academy of Sciences, \\ P. O. Box 912, Beijing 100083, China \\ ${ }^{5}$ Beijing National Laboratory of Condensed Matter Physics, Institute of Physics, Chinese Academy of Sciences, Beijing 100190, China
}

(Received 23 July 2020; revised 13 October 2020; accepted 30 October 2020; published 21 December 2020)

\begin{abstract}
Photon helicity-dependent photocurrent is measured at zero magnefic field thanks to a device based on an ensemble of (In,Ga)As/GaAs quantum dots that are embedded into a GaAs-based $p-i-n$ diode. Our main goal is to take advantage of the long electron spin-relaxation time expected in these nano-objects. In these experiments, no external magnetic field is required thanks to the use of an ultrathin magnetic $\mathrm{CoFeB} / \mathrm{MgO}$ electrode, presenting perpendicular magnetic anisotropy. We observe a clear asymmetry of the photocurrent measured under respective right and left polarized light that follows the hysteresis of the magnetic layer. The amplitude of this asymmetry at zero magnetic field decreases with increasing temperatures and can be controlled with the bias. Polarization-resolved photoluminescence is detected in parallel while the device is operated as a photodetector. This demonstrates the multifunctional capabilities of the device and gives valuable insights into the spin relaxation of the electrons in the quantum dots.
\end{abstract}

DOI: 10.1103/PhysRevMaterials.4.124603

\section{INTRODUCTION}

Intense investigations have been led on spin injection and spin properties in semiconductors in the last 20 years, in view of developing optospintronics devices [1-3] offering new functionalities based on the electron-spin degree of freedom. In particular, spin light-emitting diodes (spinLED) containing ensembles of quantum dots [4-11], where spin-polarized electrons are electrically injected, could be of particular importance for quantum information processing [12] (an electron spin confined in a semiconductor quantum dot can be used as a quantum bit) and quantum cryptography [13]. An efficient way to realize electrical spin injection into the dots consists of injecting spin-polarized electrons from a ferromagnetic injector via a tunnel barrier [14,15] (e.g., an oxide barrier like $\mathrm{MgO}$ [16-20]). This allows one to overcome the problem of conductivity mismatch between the metal and the semiconductor [21,22]. Recently, results of spin-injection processes, with electron spin aligned along the growth axis $O_{z}$ of the device, were reported in quantum wells and quantum dots without any external magnetic field (that was previously required to rotate the magnetization of the electrode along $O_{z}$ ) [23-25]. This breakthrough was realized thanks to the use of an ultrathin $\mathrm{CoFeB} / \mathrm{MgO}$ injector characterized by a strong perpendicular magnetic anisotropy (PMA) [10,23,25].

\footnotetext{
*yuan.lu@univ-lorraine.fr

†pierre.renucci@insa-toulouse.fr
}

Conversely, spin-optronic detectors or spin photodiodes, for which the amplitude of the photocurrent depends on the helicity of the detected photon and on the direction of the magnetization of the ferromagnetic (FM) electrode, may be of interest for optical telecommunications based on photon polarization [26]. This new generation of devices could be also very useful for memory-based devices with an optical reading of information stored in the ferromagnetic layer. Several works have been published dealing with such helicity-sensitive detectors based on metal-oxidesemiconductor junctions [27-30] or spin-LEDs [31-33]. There are three key issues to obtain an efficient helicity detector. Firstly, an electron spin-relaxation time in the semiconductor part is long enough such that the spin is maintained until the carrier crosses the tunnel barrier before to be collected by the ferromagnetic electrode. Secondly, the realistic device should has the ability to work at zero magnetic field. Up to now, most of the studies have been performed using external magnetic fields on the order of $1 \mathrm{~T}[28,29,31]$ to align the magnetization of the electrode along the growth direction of the structure in order to obey the condition imposed by the optical selection rules in the semiconductor [34]. Among these studies, a few of them were realized at zero external magnetic field using PMA detectors [30,33,35] or in-plane detectors $[32,36]$ under oblique incidence angle. Thirdly, it is also important to have a carrier recombination channel [31,37] that is competitive with the extraction channel from the semiconductor to the ferromagnetic electrode, in order to get an helicity asymmetry of the photocurrent under circularly polarized light in continuous-wave (cw) experiments. 


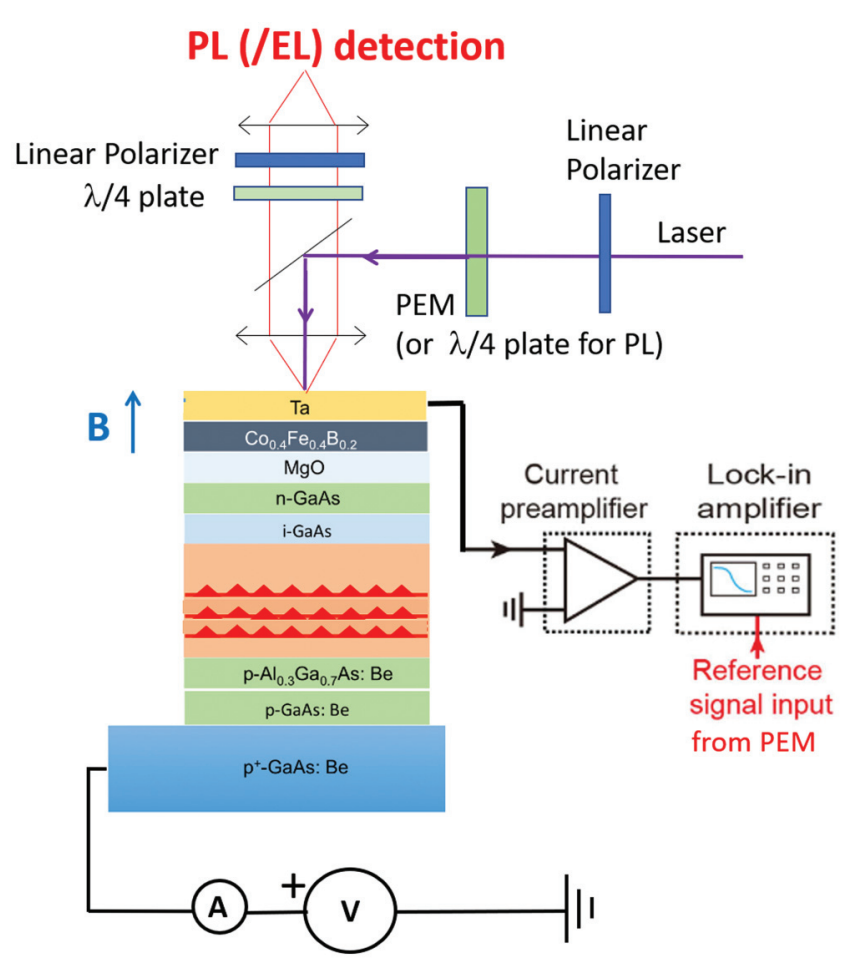

FIG. 1. Schematics of measurement setup. Spin light-emittingdiode device that can be operated either in LED mode when supplied by a voltage generator or in the photocurrent mode when excited by a circularly polarized laser. Polarization-resolved photoluminescence is detected in parallel while the device is operated in the photocurrent mode.

In this paper we propose to use $p$-doped quantum dots embedded in the active region of the spin-photodiode in order to take benefit from the long electron spin-relaxation time expected in such nano-objects [38], coupled with an ultrathin $\mathrm{CoFeB}$ magnetic electrode presenting PMA [39] to fulfill the three requirements mentioned above. Using such a hybrid system, we observe an asymmetry of photocurrent under right and left circularly polarized light (of about $0.4 \%$ at low temperature) that follows the hysteresis cycle of the magnetic layer. This is a report of a spin detector based on semiconductor quantum dots. The photocurrent asymmetry amplitude decreases with increasing temperatures and can be controlled by the bias. Moreover, as emphasized in this paper, polarization-resolved photoluminescence is detected in parallel while the device is operated as a photodetector. This simultaneous measurement of both polarized photocurrent and photoluminescence gives insights into electron-spin relaxation inside the dots when the bias is varied.

\section{SAMPLE AND EXPERIMENTAL SETUP}

The schematics of the spin-LED structure is displayed in Fig. 1. The $p-i-n$ LED device grown by molecular-beam epitaxy (MBE) contains three planes of (In, Ga)As quantum dots (QDs) embedded in the intrinsic region. The full sequence of the semiconductor structure is as following: $p+-\mathrm{GaAs}: \mathrm{Zn}$ (001) substrate $\left(p=3 \times 10^{18} \mathrm{~cm}^{-3}\right) / 300 \mathrm{~nm} p$-GaAs:Be $\left(p=5 \times 10^{18} \mathrm{~cm}^{-3}\right) / 400 \mathrm{~nm} p-\mathrm{Al}_{0.3} \mathrm{Ga}_{0.7} \mathrm{As}: \mathrm{Be}(p=5 \times$ $\left.10^{17}-5 \times 10^{18} \mathrm{~cm}^{-3}\right) / 3 \times[30-\mathrm{nm}$ undoped GaAs $(\mathrm{Be} \delta$ doping in the middle)/7 monolayers (In,Ga)As QD]/30-nm undoped GaAs/50-nm $n$-GaAs:Si $\left(n=10^{16} \mathrm{~cm}^{-3}\right)$. The Be $\delta$-doping concentration has been calibrated to yield approximatively one hole per dot. The LED surface is passivated with arsenic in the semiconductor (SC) MBE chamber and then transferred through air into a second MBE-sputtering interconnected system to grow the spin detector structure. The As capping layer is firstly desorbed at $300{ }^{\circ} \mathrm{C}$ in the MBE chamber and then the sample was transferred through ultrahigh vacuum to a sputtering chamber to grow $2.5-\mathrm{nm} \mathrm{MgO}$ layer followed by 1.1-nm $\mathrm{Co}_{0.4} \mathrm{Fe}_{0.4} \mathrm{~B}_{0.2}$ ferromagnetic layer and 5-nm Ta protection layer. Concerning the device fabrication, 300- $\mu \mathrm{m}$-diameter circular mesas were then processed using standard UV photolithography and etching techniques. Finally, the processed sample was cut into small pieces to perform rapid temperature annealing at $300{ }^{\circ} \mathrm{C}$ for $3 \mathrm{~min}$. Detailed growth conditions can be found elsewhere [23]. High resolution scanning transmission electron microscopy (HRSTEM) was performed to characterize the sample structure using a JEOL ARM200 equipped with a cold field-emission gun and a Gatan GIF Quantum Electron Energy-Loss Spectrometer (EELS). The images were recorded at $200 \mathrm{KV}$ whereas chemical maps were drawn from STEM-EELS spectrum images.

The sample was kept in a vibration-free closed-cycle $\mathrm{He}$ cryostat equipped with a superconducting coil enabling us to apply magnetic fields up to $\pm 2 \mathrm{~T}$ normal to the sample plane. The photocurrent measurements were performed under $\mathrm{cw}$ excitation with 850- and 910-nm wavelengths provided by laser diodes focused onto the sample with a typical spot diameter of $3 \mu \mathrm{m}$. The helicity of the laser diode is modulated by a photoelastic modulator at $50 \mathrm{kHz}$. The difference between the two photocurrents under circularly right and left polarized light is labeled $\Delta I_{\mathrm{ph}} . \Delta I_{\mathrm{ph}}$ is measured thanks to a transimpedance amplifier (gain $10^{5}$ ) followed by a lock-in amplifier. The bias applied to the structure is provided by a source meter that also measures the average photocurrent $I_{\mathrm{ph}}$. The positive (forward) bias corresponds to inject electrons from the metal part to the SC part, while the negative (reverse) bias corresponds to extract electrons from the SC part to the metal part. The laser power is kept below $20 \mu \mathrm{W}$ to stay in the linear regime. An original point of our setup is that the photoluminescence (PL) can be detected at the same time as the photocurrent (see Fig. 1). The PL was detected in the Faraday geometry with an aspheric lens $(f=8 \mathrm{~mm}$, numerical aperture $=0.5)$. The collected light was dispersed by a spectrometer and recorded by a cooled charge-coupled-device camera. For electroluminescence (EL) experiments, the light collection system is the same as for PL, as well as the spectral and polarizationresolved setup. The EL(PL) circular polarization degree $P_{\mathrm{c}}$ was analyzed through a $\lambda / 4$ wave plate and a linear analyzer. $P_{\mathrm{c}}$ is defined as $P_{\mathrm{c}}=\left(I^{\sigma+}-I^{\sigma-}\right) /\left(I^{\sigma+}+I^{\sigma-}\right)$ where $I^{\sigma+}$ and $I^{\sigma-}$ are the intensities of the right and left circularly polarized components of the EL/PL, respectively.

\section{STRUCTURE CHARACTERIZATION}

An atomic force microscopy (AFM) image of a freestanding (In,Ga)As QDs sample on GaAs substrate without capping 

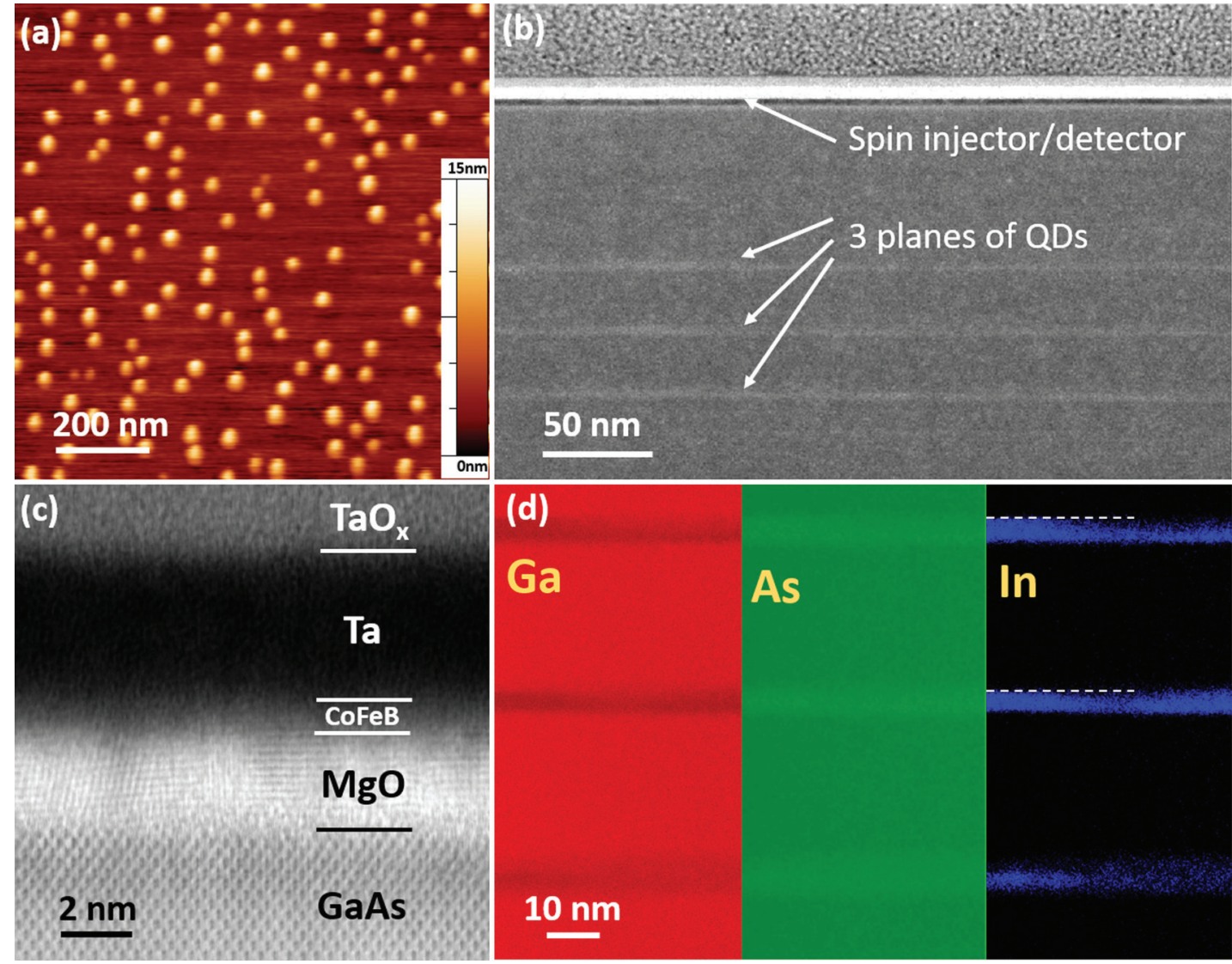

FIG. 2. (a) AFM image of the (In,Ga)As QD layer without capping with GaAs layer. The (In,Ga)As QDs have a density of $1.6 \times 10^{10} \mathrm{~cm}^{-2}$. The QDs have an average diameter of about $30 \mathrm{~nm}$ and height of about $9 \mathrm{~nm}$. (b) STEM large-scale annular dark-field (ADF) image showing three planes of QDs and homogeneous FM/SC interface. (c) STEM bright-field (BF) image showing the stack structure of spin detector. (d) EELS map showing the chemical component variation in three QD planes.

of GaAs layer is shown in Fig. 2(a). The (In,Ga)As QDs have a density of $1.6 \times 10^{10} \mathrm{~cm}^{-2}$. The average lateral dot diameter is about $30 \mathrm{~nm}$ and the height is about $9 \mathrm{~nm}$. It is important to note that the AFM image provides only an estimation of the QD morphology and density in the final device. In fact, in the device structure, the QDs are capped by the GaAs layer, which can modify the morphology and the size of QDs [40]. To clarify the QDs structure in the final device, we have performed STEM analysis combined with EELS mapping. Figure 2(b) shows the large-scale annular dark-field (ADF) STEM image of the device. Three planes of QDs can be well distinguished. Figure 2(d) displays the EELS maps for Ga, As, and In elements. It is interesting to find that the top of (In, Ga)As QD is flat with a much reduced height of about $5 \mathrm{~nm}$, as indicated by the white dashed lines. In fact, the growth of QD is performed at low temperature of $500^{\circ} \mathrm{C}$, while the growth of capping layer and top $n$-GaAs layer is carried out at high temperature of $600{ }^{\circ} \mathrm{C}$. These results indicate that the capping layer and top-layer growth condition can lead to QD shape modification via lateral migration of indium from the top of QD, which also increases the lateral diameter of QD [40].

In Fig. 2(b), the spin-detector structure deposited by sputtering appears very homogeneous on the semiconductor surface. Figure 2(c) displays the enlarged interface structure of $\mathrm{MgO} / \mathrm{CoFeB} / \mathrm{Ta}$ stacks. We can find a thin amorphous $\mathrm{GaAs}$ layer appearing at the $\mathrm{GaAs} / \mathrm{MgO}$ interface, which could be due to the large kinetic energy of the atoms reaching the surface during the sputtering growth process. The $\mathrm{MgO}$ layer shows a textured structure while $\mathrm{CoFeB}$ and $\mathrm{Ta}$ layers are almost amorphous. The top of the Ta layer is partially oxidized. The homogeneous structure ensures a high efficiency for spin injection and detection, which will be presented below.

\section{ELECTROLUMINESCENCE CHARACTERIZATION AT ZERO MAGNETIC FIELD}

The inset of Fig. 3 shows a typical polarization-resolved EL spectrum at $15 \mathrm{~K}$ and at zero magnetic field after the $\mathrm{CoFeB}$ electrode has been magnetized up to saturation along the $O_{z}$ growth axis. Remarkably, the ensemble of quantum-dot emission shows a significant circular polarization of $P_{\mathrm{c}}=$ $18 \%$ when a DC current of magnitude $160 \mu \mathrm{A}$ is applied. Thanks to the $p$ doping of the dots (one hole per dot on average), the emitting quasiparticles are positively charged excitons (trions $X^{+}$), constituted by two valence holes and one conduction electron, which are circularly polarized, contrary to neutral excitons that are linearly polarized (due to the shape anisotropy of the quantum dots) $[38,41,42]$. The 


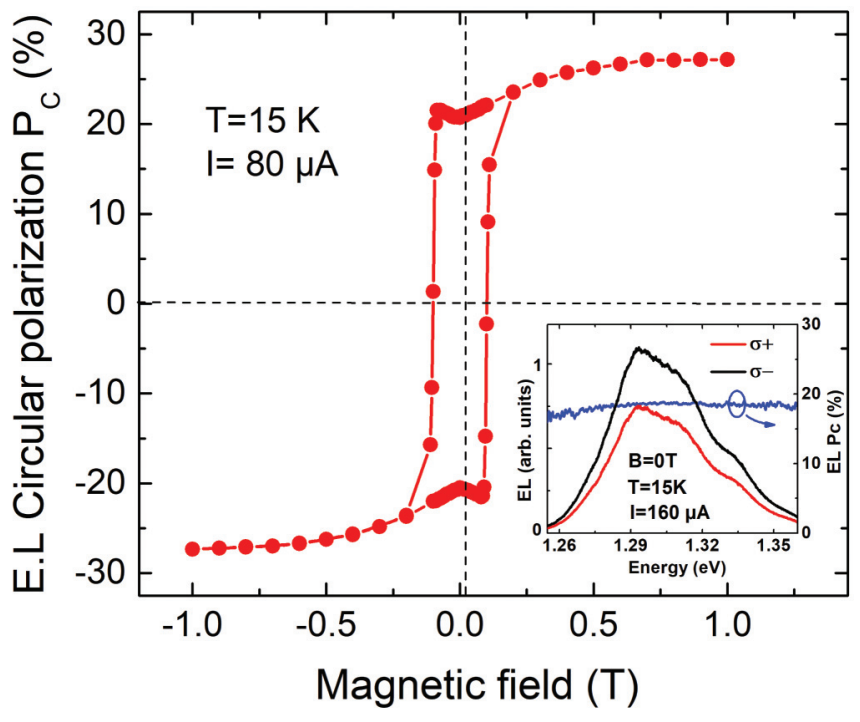

FIG. 3. EL circular polarization as a function of external magnetic field. $T=15 \mathrm{~K} . I=80 \mu \mathrm{A}$. Inset. Left axis: electroluminescence spectra of the ensemble of quantum dots. $\sigma^{+}$and $\sigma^{-}$are the right and left components (respectively). $B=0 \mathrm{~T}$. $T=15 \mathrm{~K}$. Right axis: Corresponding circular polarization $P_{\mathrm{c}}$.

resulting optical selection rules are quite straightforward: $P_{\mathrm{c}}$ is equal in the first approximation to the electron's spin polarization $P_{\mathrm{s}}\left[P_{\mathrm{s}}=\left(n^{-}-n^{+}\right) /\left(n^{-}+n^{+}\right)\right]$, where $n^{-}$and $n^{+}$ are, respectively, the number of electrons with spin down and -up in the ensemble of quantum dots, provided that the electron spin-relaxation time within the trion is longer than the electron lifetime (which is already validated for InGaAs p-doped quantum dots [38]). Long electron spin-relaxation time (a few ns) in $p$-doped quantum dots is due to the fact that the exchange interactions between the electron and the two holes with opposite spins cancel each other within the trion quasiparticle [38]. Figure 3 displays the EL circular polarization at $15 \mathrm{~K}$ as a function of the external magnetic field at a fixed bias of $V=2.2 \mathrm{~V}(I=80 \mu \mathrm{A})$. The EL polarization follows the $\mathrm{CoFeB}$ magnetic hysteresis cycle. Remarkably we find $P_{\mathrm{c}} \sim 20 \%$ at $B=0 \mathrm{~T}$. The ultrathin $\mathrm{CoFeB}$ layer presents a perpendicular magnetic anisotropy due to the interfacial anisotropy at the $\mathrm{CoFeB} / \mathrm{MgO}$ interface [39] that is responsible for this PMA hysteresis. Note that the magnetic circular dichroism (MCD) is below 1\% for this type of injector [23].

In Fig. 3, small circular polarization dips are observed when magnetic field is closed to $B=0 \mathrm{~T}$. In the $0-100 \mathrm{mT}$ range, this may be due to the electron spin-relaxation induced by the hyperfine interaction between the nuclear spins and the spin-polarized electron in the dot [43]. In this picture, this spin-relaxation mechanism is quenched when the external magnetic field is applied from 0 to about $100 \mathrm{mT}$ [38], leading to an increase of $P_{\mathrm{c}}$. For magnetic fields larger than $100 \mathrm{mT}$, we still observe a slight increase of $P_{\mathrm{c}}$. This can result from the possible circularization of the neutral exciton eigenstates [42] [the broad luminescence may include a (small) fraction of neutral excitons $X$ recombination, in addition to the $X^{+}$ trions component]. Magnetic domain alignment in the magnetic layer close to the interface may also play a role in the slight $P_{\mathrm{c}}$ increase measured in the $0.2-1 \mathrm{~T}$ range. The precise physical origin of the shape of the polarization hysteresis loop will require further investigations. However the observation of an EL circular polarization of about $20 \%$ at $B=0 \mathrm{~T}$ is a clear manifestation of a very efficient electrical spin injection without external magnetic field, and proves the high quality of the $\mathrm{CoFeB} / \mathrm{MgO} / \mathrm{GaAs}$ interface.

\section{HELICITY-DEPENDENT PHOTOCURRENT}

We then turn to photocurrent experiments under circularly polarized light. The ensemble of quantum dots is excited by a laser diode at $910 \mathrm{~nm}$ (corresponding to high-energy InGaAs dots emission/absorption) and $850 \mathrm{~nm}$ (close to the wetting layer states energy) with comparable results. Figure 4(a) shows $I-V$ characteristics of the devices in dark condition at different temperatures. Due to the $p-i-n$ junction structure, the device shows a rectified character with current injection in positive (forward) bias condition. With increasing temperature up to $132 \mathrm{~K}$, the threshold bias decreases in forward bias condition while the dark current almost keeps unchanged in reverse bias condition. Thanks to our specific setup, it is possible to detect the photocurrent and the photoluminescence signals at the same time. Figure 4(b) displays both the photocurrent and PL signals as a function of the applied bias on the device. As expected, the two signals are complementary. When a positive bias is applied to the structure (just below the bias threshold required for the appearance of EL signal), the photocurrent intensity is decreasing while by correlation the PL signal is increasing. To understand this phenomenon, we have performed a simulation of the band structure of the spin-photodiode device by using a self-consistent "1D POISSON" program [44,45]. The nominal layer thicknesses and the dopant concentrations are input parameters for the simulation. For simplification, the $\mathrm{In}_{0.3} \mathrm{Ga}_{0.7} \mathrm{As}$ QD layer has been modeled by a 9-nm-thick $\mathrm{In}_{0.3} \mathrm{Ga}_{0.7} \mathrm{As} \mathrm{QW}$ layer. Figure 5 displays the simulation of band structures. In the forward bias condition, the bands are getting flatter when the bias increases. On the contrary, for reverse bias condition, the bending of the bands increases in the depletion region of the structure, and the extraction of carriers out of the active region by tunnel effect is more efficient due to the strong electric field, leading to an increase of the photocurrent signal. At the same time, the PL signal decreases due to this escape of carriers out of the dots that constitutes an efficient nonradiative channel.

We define an asymmetry factor $A=\Delta I_{\mathrm{ph}} / I_{\mathrm{ph}}$ (where $I_{\mathrm{ph}}$ is the average photocurrent) to quantify the helicity-dependent photocurrent. In Fig. 4(d), at $V=+0.4 \mathrm{~V}$, one can observe that the asymmetry factor $A$ is about $0.4 \%$ at zero magnetic field and $T=30 \mathrm{~K}$ after the $\mathrm{CoFeB}$ layer has been magnetized at saturation along the $O_{z}$ growth axis. Moreover, the factor $A$ follows the hysteresis cycle of the ultrathin $\mathrm{CoFeB}$ magnetic layer. The observed coercive field of about $100 \mathrm{mT}$ is comparable to the one measured by EL in Fig. 3. Remarkably, the helicity-dependent photocurrent can be modified by applying an external bias [Fig. 4(e)]. Note that this bias dependence of $A$ allows us to rule out a possible artifact due to magnetic circular dichroism (a flat background independent of the applied bias that may be due to a small MCD has been properly subtracted). It appears that $A$ increases 

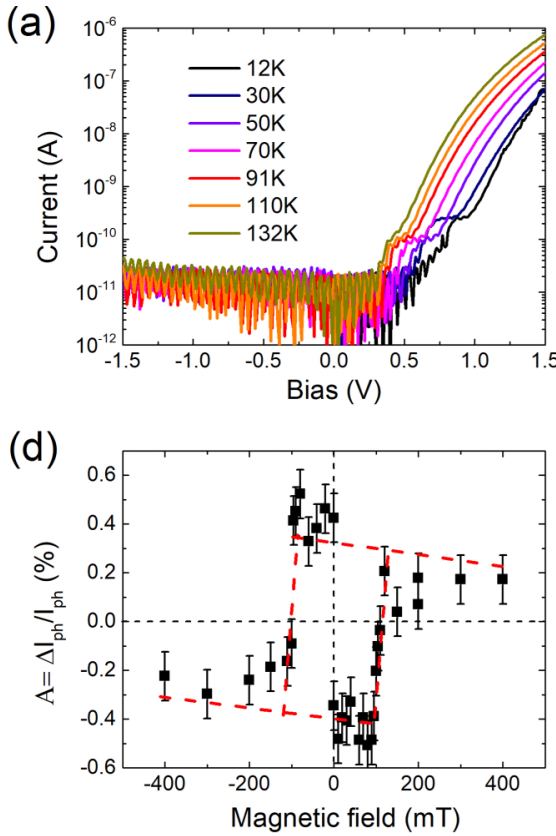
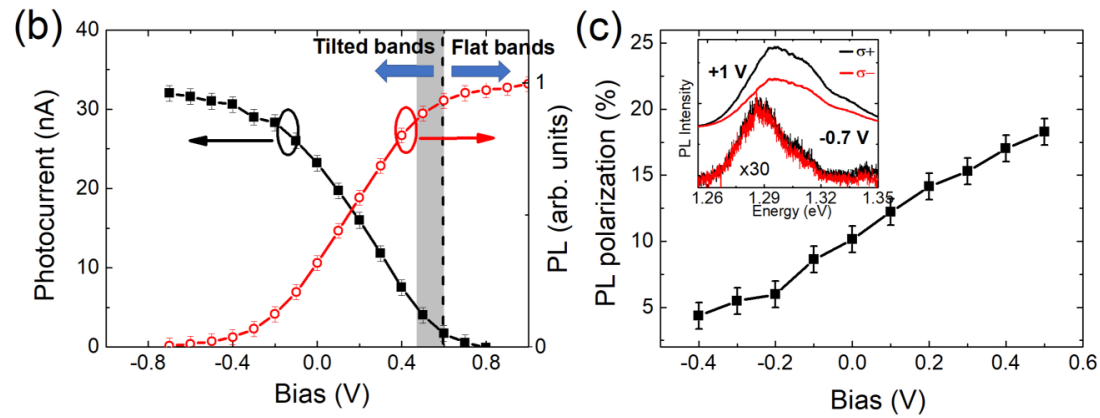

(e)

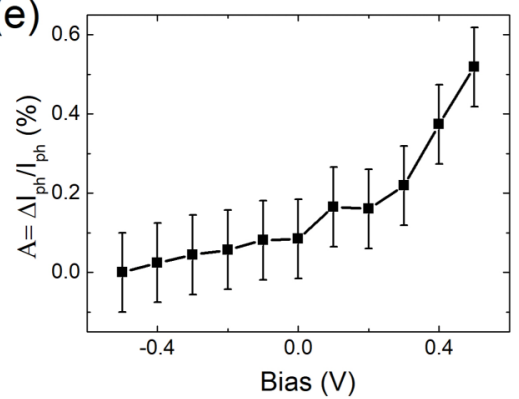

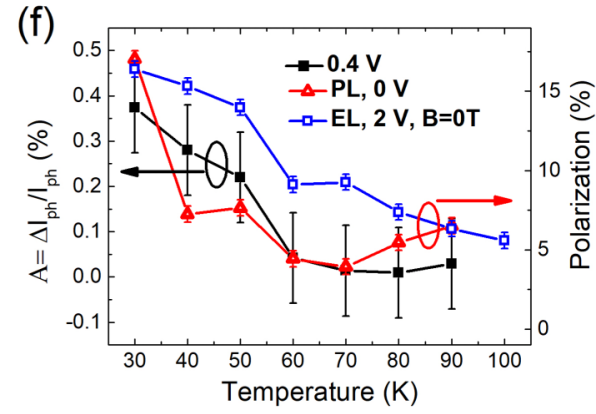

FIG. 4. (a) $I-V$ characteristics of the device at different temperatures. (b) Photocurrent (black squares, left axis) and photoluminescence signals (open red circles, right axis) as a function of bias. $\lambda_{\text {laser }}=850 \mathrm{~nm}$. The gray zone indicates the bias window for which the photocurrent asymmetry $A$ displayed in (e) is maximal. (c) Circular polarization of the photoluminescence as a function of the bias. $\lambda_{\text {laser }}=850 \mathrm{~nm}$. $T=10 \mathrm{~K} . B=0 \mathrm{~T}$. Inset: $\sigma+$ (black line) and $\sigma-$ (red line) components of the luminescence for $V=+1 \mathrm{~V}$ and $-0.7 \mathrm{~V}$. (d) Photocurrent helicity asymmetry $A$ as a function of the external magnetic field. $\lambda_{\text {laser }}=910 \mathrm{~nm}, V=+0.4 \mathrm{~V}, T=30 \mathrm{~K}$. (e) Photocurrent helicity asymmetry $A$ as a function of applied bias at zero external magnetic field. $\lambda_{\text {laser }}=850 \mathrm{~nm}$. $T=30 \mathrm{~K}$. (f) Photocurrent helicity asymmetry $A$ (black squares, left axis) as a function of temperature at fixed bias. $\lambda_{\text {laser }}=850 \mathrm{~nm}, V=+0.4 \mathrm{~V}, B=0 \mathrm{~T}$. PL circular polarization (red triangles, right axis). $\lambda_{\text {laser }}=850 \mathrm{~nm}, V=0 \mathrm{~V}, B=0 \mathrm{~T}$. EL circular polarization (open blue squares, right axis) as a function of temperature. $V=+2.0 \mathrm{~V}$, $I=54 \mu \mathrm{A}, B=0 \mathrm{~T}$.

with the applied bias, in the forward regime for the $p-i-n$ junction (in the flat-band regime slightly before EL emission starts). We can see in Fig. 4(b) that the bias range (marked by gray zone) for which $A$ is maximum corresponds to a relatively weak photocurrent amplitude and a large PL inten-

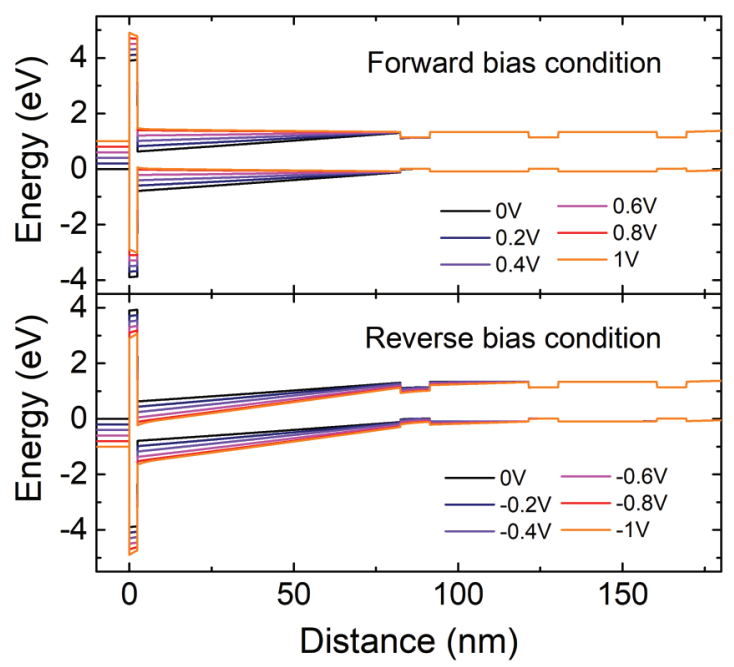

FIG. 5. Energy band diagram simulations using a one-dimensional Poisson-Schrödinger solver for the forward and reverse bias conditions at $10 \mathrm{~K}$. sity. In this regime, radiative recombination in the quantum dots constitutes a strongly competing channel to photocurrent extraction.

Two main reasons could explain the large increase of $A$ under forward bias. First, a channel competing with respect to the extraction of carriers from the dots (or localized states in the wetting layer) towards the magnetic electrode is required to obtain a sizable photocurrent asymmetry [31,37]. Indeed, in the absence of any competing channel (PL, interface recombination, ...), cw excitation would lead to helicity-dependent spin accumulation at the interface with the $\mathrm{MgO}$ barrier but, however, zero photocurrent asymmetry. In our case, the radiative recombination channel in the dots could constitute a competing channel that becomes more efficient under forward bias, and would lead to a larger value of the photocurrent asymmetry. The second possible reason is that the trion/exciton population rate in the QDs could depend on the applied bias. Some insight about this dependence can be obtained by polarization-resolved PL experiments under bias [Fig. 4(c)]. As expected, the spectrum at $-0.7 \mathrm{~V}$ is redshifted compared to the one at $+1 \mathrm{~V}$, due to quantum-confined Stark effect [46]. This can be also seen from the simulated band structure (Fig. 5) that the negative bias can change the band structure in the active region. The spectrum observed at $-0.7 \mathrm{~V}$ is less broad at high energy, because of the easiest escape out of the dots of electrons with high energy by tunneling effect from quantum dots under reverse bias. The PL circular 
polarization of the ensemble of dots (integrated on the whole spectrum) increases strongly from about $4 \%$ to about $18 \%$ when the bias varies from -0.6 to $+0.6 \mathrm{~V}$. We believe that this can be explained by the bias-induced change of trion/exciton population ratio within the ensemble of dots. Indeed, a positive bias favors the presence of one resident hole in the dot, and thus the photogeneration of trions (which are circularly polarized) instead of neutral excitons (whose eigenstates are linearly-polarized). The corresponding change in the trion/exciton population ratio yields an increase of the PL circular polarization. This polarization increase will also contribute to the larger value of the photocurrent asymmetry obtained at positive bias [Fig. 4(e)].

Finally, we have also studied the dependence of the photocurrent asymmetry $A=\Delta I_{\mathrm{ph}} / I_{\mathrm{ph}}$ as a function of temperature [Fig. 4(f)]. A clear drop is observed when the temperature varies from 30 to $90 \mathrm{~K}$. Above $90 \mathrm{~K}$, the signal disappears. The trend is the same for the EL circular polarization and the PL circular polarization [Fig. 4(f)]. Note that this temperature dependence also allows us to rule out artificial effect coming from MCD. Two possibilities could explain the temperature variation of photocurrent asymmetry. Firstly, this behavior is probably due to a phonon-induced spin-relaxation mechanism, as clearly evidenced in InAs/GaAs quantum dots $[47,48]$. The second possibility is linked to the average doping per quantum dot, which varies significantly when the temperature increases. This variation of QD doping was clearly identified in the temperature-dependence measurements of intersublevel absorption [49]. It has also strong consequences on the amplitude of the measured EL circular polarization since the QD eigenstates change with the doping [Fig. 4(f)] [11]. It is well known that the exciton eigenstates of undoped InGaAs quantum dots are linearly polarized as a consequence of the anisotropic exchange interaction [50]. As a consequence, the spin polarization of photocurrent excited by circular polarized light will be much reduced when the temperature increases, as well as the photocurrent asymmetry detected by the spin detector.

\section{CONCLUSION}

Thanks to the use of an ultrathin magnetic layer of CoFeB that presents perpendicular magnetic anisotropy, and $p$-doped (In, Ga)As quantum dots, we have evidenced at zero external magnetic field an asymmetry of photocurrent under right and left polarized light that follows the hysteresis cycle of the magnetic electrode. The amplitude of this asymmetry at zero magnetic field is about $0.4 \%$ at low temperature, and can be controlled by the bias. It paves the way for versatile devices operating at zero magnetic field with two functionalities (helicity-dependent photodetector and circularly polarized light source). Note that the use of an ultrathin $\mathrm{CoFeB}$ electrode of a few atomic layers is a strong advantage compared to thicker stacks $[35,48,51]$ which provide the required perpendicular magnetic anisotropy, because it minimizes the light absorption in the magnetic layer. This is a crucial point for the realization of sensitive photodetectors.

\section{ACKNOWLEDGMENTS}

We thank Michel Hehn for his help to develop the growth of ultrathin $\mathrm{CoFeB}$ layer by sputtering. F.C, P.R., and H.C acknowledge the Grant NEXT No. ANR-10-LABX-0037 in the framework of the "Programme des Investissements d'Avenir." This work was also supported by the joint French National Research Agency (ANR)-National Natural Science Foundation of China (NSFC) SISTER project (Grants No. ANR-11-IS10-0001 and No. NNSFC 61161130527) and ENSEMBLE project (Grants No. ANR-14-0028-01 and No. NNSFC 61411136001). Experiments were performed using equipment from the platform TUBE Davm funded by FEDER (EU), ANR, the Region Lorraine and Grand Nancy. X.M acknowledges the Institut Universitaire de France. X.F.H acknowledges the support of NSFC (Grant No. 51620105004).
[1] R. Fiederling, M. Keim, G. Reuscher, W. Ossau, G. Schmidt, A. Waag, and L. W. Molenkamp, Injection and detection of a spinpolarized current in a light-emitting diode, Nature (London) 402, 787 (1999).

[2] Y. Ohno, D. K. Young, B. Beschoten, F. Matsukura, H. Ohno, and D. D. Awschalom, Electrical spin injection in a ferromagnetic semiconductor heterostructure, Nature (London) 402, 790 (1999).

[3] M. Lindemann, G. Xu, T. Pusch, R. Michalzik, M. R. Hofmann, I. Zutic, and N. C. Gerhardt., Ultrafast spin-lasers, Nature (London) 568, 212 (2019).

[4] W. Löffler, D. Tröndle, J. Fallert, H. Kalt, D. Litvinov, D. Gerthsen, J. Lupaca-Schombert, T. Passow, B. Daniel, J. Kvietkova, M. Grün, C. Klingshirn, and M. Hetterich, Electrical spin injection from $\mathrm{ZnMnSe}$ into InGaAs quantum wells and quantum dots, Appl. Phys. Lett. 88, 062105 (2006).

[5] C. H. Li, G. Kioseoglou, O. M. J. van't Erve, M. E. Ware, D. Gammon, R. M. Stroud, B. T. Jonker, R. Mallory, M. Yasar, and A. Petrou, Electrical spin pumping of quantum dots at room temperature, Appl. Phys. Lett. 86, 132503 (2005).
[6] G. Itskos, E. Harbord, S. K. Clowes, E. Clarke, L. F. Cohen, R. Murray, P. Van Dorpe, and W. Van Roy, Oblique Hanle measurements of InAs/GaAs quantum dot spin-light emitting diodes, Appl. Phys. Lett. 88, 022113 (2006).

[7] L. Lombez, P. Renucci, P. F. Braun, H. Carrère, X. Marie, T. Amand, B. Urbaszek, J. L. Gauffier, P. Gallo, T. Camps, A. Arnoult, C. Fontaine, C. Deranlot, R. Mattana, H. Jaffrès, J.-M. George, and P. H. Binh, Electrical spin injection into p-doped quantum dots through a tunnel barrier, Appl. Phys. Lett. 90, 081111 (2007).

[8] W. Löffler, M. Hetterich, C. Mauser, S. Li, T. Passow, and H. Kalt, Parallel preparation of highly spin-polarized electrons in single InAs/GaAs quantum dots, Appl. Phys. Lett. 90, 232105 (2007).

[9] P. Asshoff, G. Wüst, A. Merz, D. Litvinov, D. Gerthsen, H. Kalt, and M. Hetterich, Nuclear spin polarization in single selfassembled In $0.3 \mathrm{Ga} 0.7$ As quantum dots by electrical spin injection, Phys. Rev. B 84, 125302 (2011).

[10] F. Cadiz, A. Djeffal, D. Lagarde, A. Balocchi, B. Tao, B. Xu, S. Liang, M. Stoffel, X. Devaux, H. Jaffrès, J.-M. George, M. 
Hehn, S. Mangin, H. Carrère, X. Marie, T. Amand, X. Han, Z. Wang, and B. Urbaszek. Y. Lu, and P. Renucci, Electrical initialization of electron and nuclear spins in a single quantum dot at zero magnetic field, Nano Lett. 18, 2381 (2018).

[11] A. E. Giba, X. Gao, M. Stoffel, X. Devaux, B. Xu, X. Marie, P. Renucci, H. Jaffrès, J.-M. George, G. Cong, Z. Wang, H. Rinnert, and Y. Lu, Spin Injection and Relaxation in P-Doped (In,Ga)As/GaAs Quantum-Dot Spin Light-Emitting Diodes at Zero Magnetic Field, Phys. Rev. Appl. 14, 034017 (2020).

[12] Semiconductor Quantum Bits, edited by F. Henneberger and O. Benson (Pan Stanford Publ., World Scientific, Singapore, 2009).

[13] R. M. Stevenson, R. J. Young, P. Atkinson, K. Cooper, D. A. Ritchie, and A. J. Shields, A semiconductor source of triggered entangled photon pairs, Nature (London) 439, 179 (2006).

[14] A. T. Hanbicki, O. M. J. van't Erve, R. Magno, G. Kioseoglou, C. H. Li, B. T. Jonker, G. Itskos, R. Mallory, M. Yasar, and A. Petrou, Analysis of the transport process providing spin injection through an Fe/AlGaAs Schottky barrier, Appl. Phys. Lett. 82, 4092 (2003).

[15] J. Strand, X. Lou, C. Adelmann, B. D. Schultz, A. F. Isakovic, C. J. Palmstrom, and P. A. Crowell, Electron spin dynamics and hyperfine interactions in Fe/Al $0.1 \mathrm{Ga} 0.9 \mathrm{As} / \mathrm{Ga}$ As spin injection heterostructures, Phys. Rev. B 72, 155308 (2005).

[16] X. Jiang, R. Wang, R. M. Shelby, R. M. Macfarlane, S. R. Bank, J. S. Harris, and S. S. P. Parkin, Highly Spin-Polarized Room-Temperature Tunnel Injector for Semiconductor Spintronics using $\mathrm{MgO}(100)$, Phys. Rev. Lett. 94, 056601 (2005).

[17] Y. Lu, V. G. Truong, P. Renucci, M. Tran, H. Jaffrès, C. Deranlot, J.-M. George, A. Lemaître, Y. Zheng, D. Demaille, P.H. Binh, T. Amand, and X. Marie, MgO thickness dependence of spin injection efficiency in spin-light emitting diodes, Appl. Phys. Lett. 93, 152102 (2008).

[18] V. G. Truong, P.-H. Binh, P. Renucci, M. Tran, Y. Lu, H. Jaffrès, J.-M. George, C. Deranlot, A. Lemaître, T. Amand, and X. Marie, High speed pulsed electrical spin injection in spin-light emitting diode, Appl. Phys. Lett. 94, 141109 (2009).

[19] P. Barate, S. Liang, T. T. Zhang, J. Frougier, M. Vidal, P. Renucci, X. Devaux, B. Xu, H. Jaffrès, J. M. George, X. Marie, M. Hehn, S. Maning, Y. Zheng, T. Amand, B. Tao, X. F. Han, Z. Wang, and Y. Lu, Electrical spin injection into InGaAs/GaAs quantum wells: A comparison between $\mathrm{MgO}$ tunnel barriers grown by sputtering and molecular beam epitaxy methods, Appl. Phys. Lett. 105, 012404 (2014).

[20] P. Barate, S. H. Liang, T. T. Zhang, J. Frougier, B. Xu, P. Schieffer, M. Vidal, H. Jaffrès, B. Lépine, S. Tricot, F. Cadiz, T. Garandel, J. M. George, T. Amand, X. Devaux, M. Hehn, S. Mangin, B. Tao, X. F. Han, Z. G. Wang, X. Marie, Y. Lu, and $\mathrm{P}$. Renucci, Bias dependence of the electrical spin injection into $\mathrm{GaAs}$ from $\mathrm{Co}-\mathrm{Fe}-\mathrm{B} / \mathrm{MgO}$ Injectors with Different $\mathrm{MgO}$ Growth Processes, Phys. Rev. Appl. 8, 054027 (2017).

[21] G. Schmidt, D. Ferrand, L. W. Molenkamp, A. T. Filip, and B. J. van Wees, Fundamental obstacle for electrical spin injection from a ferromagnetic metal into a diffusive semiconductor, Phys. Rev. B 62, R4790 (2000).

[22] A. Fert and H. Jaffrès, Conditions for efficient spin injection from a ferromagnetic metal into a semiconductor, Phys. Rev. B 64, 184420 (2001).

[23] S. H. Liang, T. T. Zhang, P. Barate, J. Frougier, M. Vidal, P. Renucci, B. Xu, H. Jaffrès, J.-M. George, X. Devaux, M. Hehn,
X. Marie, S. Mangin, H. X. Yang, A. Hallal, M. Chshiev, T. Amand, H. F. Liu, D. P. Liu, X. F. Han, Z. G. Wang, and Y. Lu, Large and robust electrical spin injection into GaAs at zero magnetic field using an ultrathin $\mathrm{CoFeB} / \mathrm{MgO}$ injector, Phys. Rev. B 90, 085310 (2014).

[24] B. S. Tao, P. Barate, J. Frougier, P. Renucci, B. Xu, A. Djeffal, H. Jaffrès, J.-M. George, X. Marie, S. Petit-Watelot, S. Mangin, X. F. Han, Z. G. Wang, and Y. Lu, Electrical spin injection into GaAs based light emitting diodes using perpendicular magnetic tunnel junction-type spin injector, Appl. Phys. Lett. 108, 152404 (2016).

[25] B. Tao, P. Barate, X. Devaux, P. Renucci, J. Frougier, A. Djeffal, S. Liang, B. Xu, M. Hehn, H. Jaffrès, J. M. George, X. Marie, S. Mangin, X. Han, Z. Wang, and Y. Lu, Atomic-scale understanding of high thermal stability of the $\mathrm{Mo} / \mathrm{CoFeB} / \mathrm{MgO}$ spin injector for spin-injection in remanence, Nanoscale 10, 10213 (2018).

[26] R. Farshchi, M. Ramsteiner, J. Herfort, A. Tahraoui, and H. T. Grahn, Optical communication of spin information between light emitting diodes, Appl. Phys. Lett. 98, 162508 (2011).

[27] A. Hirohata, Y. B. Xu, C. M. Guertler, and J. A. C. Bland, Spin-dependent electron transport in NiFe/GaAs Schottky barrier structures, J. Appl. Phys. 87, 4670 (2000).

[28] T. Taniyama, G. Wastlbauer, A. Ionescu, M. Tselepi, and J. A. C. Bland, Spin-selective transport through Fe/AlOx/GaAs (100) interfaces under optical spin orientation, Phys. Rev. B 68 , 134430 (2003)

[29] C. Rinaldi M. Cantoni, D. Petti, A. Sottocorno, M. Leone, N. M. Caffrey, S. Sanvito, and R. Betacco, Ge-based spin-photodiodes for room-temperature integrated detection of photon helicity, Adv. Mater. 24, 3037 (2012).

[30] A. Djeffal, F. Cadiz, M. Stoffel, D. Lagarde, X. Gao, H. Jaffrès, X. Devaux, S. Migot, X. Marie, H. Rinnert, S. Mangin, J.-M George, P. Renucci, and Y. Lu, Co-Fe-B/MgO/Ge Spin Photodiode Operating at Telecommunication Wavelength with Zero Applied Magnetic Field, Phys. Rev. Appl. 10, 044049 (2018).

[31] P. Renucci, V. G. Truong, H. Jaffrès, L. Lombez, P. H. Binh, T. Amand, J. M. George, and X. Marie, Spin-polarized electroluminescence and spin-dependent photocurrent in hybrid semiconductor/ferromagnetic heterostructures: An asymmetric problem, Phys. Rev. B 82, 195317 (2010).

[32] L. Zhu, W. Huang, P. Renucci, X. Marie, Y. Liu, Y. Li, Q. Wu, Y. Zhang, B. Xu, Y. Lu, and Y. Chen, Angular dependence of the spin photocurrent in a $\mathrm{Co}-\mathrm{Fe}-\mathrm{B} / \mathrm{MgO} / \mathrm{n}-\mathrm{i}-\mathrm{p}$ GaAs quantumwell structure, Phys. Rev. Appl. 8, 064022 (2017).

[33] X. Xue, L. Zhu, W. Huang, X. Marie, P. Renucci, Y. Liu, Y. Zhang, X. Zeng, J. Wu, B. Xu, Z. Wang, Y. Chen, W. Zhang, and Y. Lu, Comparison of spin photocurrent in devices based on in-plane or out-of-plane magnetized $\mathrm{CoFeB}$ spin detectors, Phys. Rev. B 100, 045417 (2019).

[34] F. Meier and B. P. Zakharchenia, Optical Orientation (NorthHolland, Amsterdam, 1984).

[35] S. Hövel, N. C. Gerhardt, M. R. Hofmann, F.-Y. Lo, D. Reuter, A. D. Wieck, E. Schuster, W. Keune, H. Wende, O. Petracic, and K. Westerholt, Electrical detection of photoinduced spins both at room temperature and in remanence, Appl. Phys. Lett. 92, 242102 (2008)

[36] R. C. Roca, N. Nishizawa, K. Nishibayashi, and H. Munekata, Investigation of helicity-dependent photocurrent at room temperature from a $\mathrm{Fe} / \mathrm{x}-\mathrm{AlO} x / \mathrm{p}-\mathrm{GaAs}$ Schottky junction 
with oblique surface, Jpn. J. Appl. Phys. 56, 04CN05 (2017).

[37] A. F. Isakovic, D. M. Carr, J. Strand, B. D. Schultz, C. J. Palmstrøm, and P. A. Crowell, Optically pumped transport in ferromagnet-semiconductor Schottky diodes (invited), J. Appl. Phys. 91, 7261 (2002).

[38] P.-F. Braun, X. Marie, L. Lombez, B. Urbaszek, T. Amand, P. Renucci, V. K. Kalevich, K. V. Kavokin, O. Krebs, P. Voisin, and Y. Masumoto, Direct Observation of the Electron Spin Relaxation Induced by Nuclei in Quantum Dots, Phys. Rev. Lett. 94, 116601 (2005).

[39] S. Ikeda, K. Miura, H. Yamamoto, K. Mizunuma, H. D. Gan, M. Endo, S. Kanai, J. Hayakawa, F. Matsukura, and H. Ohno, A perpendicular-anisotropy $\mathrm{CoFeB}-\mathrm{MgO}$ magnetic tunnel junction, Nat. Mater. 9, 721 (2010).

[40] E. C. Weiner, R. Jakomin, D. N. Micha, H. Xie, P.-Y. Su, L. D. Pinto, M. P. Pires, F. A. Ponce, and P. L. Souza, Effect of capping procedure on quantum dot morphology: Implications on optical properties and efficiency of InAs/GaAs quantum dot solar cells, Sol. Energy Mater. Sol. Cells 178, 240 (2018).

[41] M. Bayer, A. Kuther, A. Forchel, A. Gorbunov, V. B. Timofeev, F. Schäfer, J. P. Reithmaier, T. L. Reinecke, and S. N. Walck, Electron and Hole g Factors and Exchange Interaction from Studies of the Exciton Fine Structure in In 0.60 Ga 0.40 As Quantum Dots, Phys. Rev. Lett. 82, 1748 (1999).

[42] M. Paillard, X. Marie, P. Renucci, T. Amand, A. Jbeli, and J. M. Gérard, Spin Relaxation Quenching in Semiconductor Quantum Dots, Phys. Rev. Lett. 86, 1634 (2001).

[43] I. A. Merkulov, Al. L. Efros, and M. Rosen, Electron spin relaxation by nuclei in semiconductor quantum dots, Phys. Rev. B 65, 205309 (2002).
[44] G. L. Snider, I. H. Tan, and E. L. Hu, Electron states in mesaetched one-dimensional quantum well wires, J. Appl. Phys. 68, 2849 (1990).

[45] I. H. Tan, G. L. Snider, L. D. Chang, and E. L. Hu, A selfconsistent solution of Schrödinger-Poisson equations using a nonuniform mesh, J. Appl. Phys. 68, 4071 (1990).

[46] A. J. Bennett, R. B. Patel, J. Skiba-Szymanska, C. A. Nicoli, I. Farrer, D. A. Ritchie, and A. J. Shields, Giant Stark effect in the emission of single semiconductor quantum dots, Appl. Phys. Lett. 97, 031104 (2010).

[47] A. Tackeuchi, R. Ohtsubo, M. Murayama, and T. Takagahara, Spin relaxation dynamics in highly uniform InAs quantum dots, Appl. Phys. Lett. 84, 3576 (2004).

[48] L. Grenet, M. Jamet, P. Noé, V. Calvo, J. M. Hartmann, L. E. Nistor, B. Rodmacq, S. Auffret, P. Warin, and Y. Samson, Spin injection in silicon at zero magnetic field, Appl. Phys. Lett. 94, 032502 (2009).

[49] F. Bras, P. Boucaud, S. Sauvage, and G. Fishman, Temperature dependence of intersublevel absorption in InAs/GaAs selfassembled quantum dots, Appl. Phys. Lett. 80, 4620 (2002).

[50] M. Bayer, G. Ortner, O. Stern, A. Kuther, A. A. Gorbunov, A. Forchel, P. Hawrylak, S. Fafard, K. Hinzer, T. L. Reinecke, S. N. Walck, J. P. Reithmaier, F. Klopf, and F. Schäfer, Fine structure of neutral and charged excitons in self-assembled In(Ga)As/(Al)GaAs quantum dots, Phys. Rev. B 65, 195315 (2002).

[51] J. Zarpellon, H. Jaffrès, J. Frougier, C. Deranlot, J. M. George, D. H. Mosca, A. Lemaître, F. Freimuth, Q. H. Duong, P. Renucci, and X. Marie, Spin injection at remanence into III-V spin light-emitting diodes using $(\mathrm{Co} / \mathrm{Pt})$ ferromagnetic injectors, Phys. Rev. B 86, 205314 (2012). 